\title{
THE EFFECT OF CHEMICAL FUNCTIONALIZATION ON MECHANICAL PROPERTIES OF NANOTUBE/POLYMER COMPOSITES
}

\author{
G.M. Odegard*, S.J.V. Frankland ${ }^{\dagger}$, T.S. Gates ${ }^{\dagger}$ \\ NASA Langley Research Center \\ Hampton, Virginia
}

\begin{abstract}
$\underline{\text { Abstract }}$
The effects of the chemical functionalization of a carbon nanotube embedded in a nanotube/polyethylene composite on the bulk elastic properties are presented. Constitutive equations are established for both functionalized and nonfunctionalized nanotube composites systems by using an equivalent-continuum modeling technique. The elastic properties of both composites systems are predicted for various nanotube lengths, volume fractions, and orientations. The results indicate that for the specific composite material considered in this study, most of the elastic stiffness constants of the functionalized composite are either less than or equal to those of the non-functionalized composite.
\end{abstract}

\section{Introduction}

Carbon nanotube-reinforced polymer composites have the potential to provide order-of-magnitude increases in strength and stiffness relative to typical carbon-fiber reinforced polymeric composites. ${ }^{1}$ The remarkable property-to-weight ratio of these materials makes them ideal candidates for aerospace-related structural applications. The mechanical behavior of nanotube/polymer composites depends not only on the individual properties of the polymer and the nanotubes, but also on the nanotube/polymer interaction.

This polymer/nanotube interaction is assumed to be confined to a cylindrical region surrounding the nanotube and extending radially away from the exterior of the tube. The strength of this nanotube/polymer interaction is defined by the ability to transfer mechanical load between the nanotube and

* Staff Scientist, National Institute of Aerospace. Member, AIAA

${ }^{\dagger}$ Staff Scientist, National Institute of Aerospace.

\$ Senior Materials Research Engineer, Mechanics and Durability Branch. Associate Fellow, AIAA.

Copyright (C) 2003 by the American Institute of Aeronautics and Astronautics, Inc. No copyright is asserted in the United States under Title 17, U.S. Code. The U.S. Government has a royalty-free license to exercise all rights under the copyright claimed herein for Governmental Purposes. All other rights are reserved by the copyright owner. polymer and has been a matter of significant discussion in the literature..$^{2-14}$ In general, two basic approaches have been proposed to improve the strength of the interaction. The first approach involves forming a strong, non-bonded interaction with the nanotube without modifying the nanotube structure. This approach assumes a chemical strengthening of the nonbonded interactions with the nanotube ${ }^{15}$ and the improvement of the mechanical connection with the nanotube; for example, by wrapping a large polymer molecule around the nanotube. ${ }^{16,17}$ The second approach requires the formation of a chemical covalent bond between the nanotube and polymer directly, also known as functionalization. There is reasonable evidence for the presence of such nanotube/polymer covalent bonds. ${ }^{18,19}$ Some studies have indicated that functionalization can occur through chemical bonds added to the nanotube sidewalls. ${ }^{3-8}$ Addition of small organic groups to nanotube sidewalls has been reported via in situ-generated diazonium compounds ${ }^{4}$ and by fluorination of nanotubes ${ }^{5,6}$ followed by alkylation. Functionalization can also occur through oxidizing the nanotube sample to induce the formation hydroxyl or carboxylic acid groups at surface defects on the nanotube. ${ }^{9-14}$ This type of functionalization can occur with co-polymers, ${ }^{9-11}$ proteins, ${ }^{12}$ organosilanes, ${ }^{13}$ and metal catalysts. ${ }^{14}$ It is known that covalent bonding affects the properties of the nanotube itself because the formation of a chemical bond with carbon atoms in a nanotube interrupts the $\mathrm{sp}^{2}$ hybridization of the nanotube, thereby forming a site that is closer to an $\mathrm{sp}^{3}$ hybridized carbon. ${ }^{20,21}$ However, analysis and tests have not provided a description of how functionalization affects the overall mechanical properties of the composite. A recent simulation study by one of the authors has predicted that for carbon nanotube/polyethylene composites there is at least a one order-of-magnitude increase in the strength for composites with covalent bonding between the nanotube and adjacent polymer molecules, relative to systems without the covalent bonds. ${ }^{22}$ However, the hybridization change may weaken the chemical bonds in the vicinity of the functionalization. The effect should be manifested in the material elastic constants. $^{15,16}$ It is proposed therefore, that hierarchal 
analysis and subsequent predictions of macroscopic, elastic behavior may provide additional insights into the relative merits of nanotube functionalization and the relationship of functionalization to nanotube/polymer load transfer and strength.

In the present paper, constitutive models are developed for nanotube/polyethylene composites, with and without functionalization, to predict the effect of functionalization on the elastic mechanical stiffness properties. The constitutive models are established using a recently developed, hierarchal modeling method for predicting the elastic properties of nanotube/polymer composites. ${ }^{23}$ The constitutive models are then used to establish equivalentcontinuum models of the representative volume elements (RVEs) of the functionalized and nonfunctionalized composites. The equivalent-continuum model is then combined with a micromechanical analysis to predict Young's modulus and the shear modulus, for various nanotube lengths, volume fractions, and orientations. Results from these analyses are quantified and the predicted properties of the functionalized and non-functionalized composite systems are compared.

\section{Equivalent-Continuum Modeling}

The constitutive model of the composite material, required by the equivalent-continuum method, uses an equilibrium molecular structure obtained with a molecular dynamics (MD) simulation. ${ }^{23}$ Using a hierarchical modeling scheme, the equivalentcontinuum modeling technique is then used to predict the bulk mechanical behavior of nano-structured materials. $^{24}$ In summary, the hierarchical model consists of three major steps. First, a suitable representative volume element (RVE) of the nanostructured material is chosen based on the geometry of the molecular model. The RVE of a typical nanostructured material is on the nanometer length scale, therefore, the material of the RVE is not continuous, but is an assemblage of many atoms. Interaction of these atoms is described in terms of molecularmechanics force constants, which are known for most atomic structures. ${ }^{25}$ Second, an equivalent-truss model of the RVE is developed as an intermediate step to link the molecular and equivalent-continuum models. Each atom in the molecular model is represented by a pin-joint, and each truss element represents an atomic bonded or non-bonded interaction. The values of the modulus of the truss elements are determined using the molecular-mechanics force constants. Therefore, the total molecular potential energy of the molecular model and the strain energy of the equivalent-truss are equal for the same loading conditions. Finally, an equivalent-continuum model of the RVE is developed in which the total strain energy in the molecular and equivalent-continuum models, under identical loading conditions, is equal. The effective mechanical properties, or the effective geometry, of the equivalentcontinuum is then determined from equating strain energies. Each step of the implementation of this method for a functionalized and non-functionalized nanotube/polyethylene composite is described below. Further details of the modeling method may be found in the previously cited references.

\section{$\underline{\text { Molecular model }}$}

MD simulations were used to determine the equilibrium structures of the functionalized and nonfunctionalized nanotube/polyethylene composites (left side of Figs. 1 and 2). For both systems, the starting configuration for the MD simulation was a crystalline polyethylene matrix containing a $(10,10)$ nanotube of radius $6.78 \AA$. The matrix contained 176 chains of 42 $\mathrm{CH}_{2}$ units. The nanotube and polymer chains were replicated across the periodic boundaries of the simulation cell, making them infinitely long. In the functionalized composite, two polymer chains were attached to six carbons of the nanotube by chemical linkages consisting of $2 \mathrm{CH}_{2}$ groups. The details of the linkages are shown in Fig. 3. In the MD simulations, all the covalent chemical bonds in the composites were modeled with the hydrocarbon potential developed by Brenner, et $a l .{ }^{26}$ The non-bonded interactions between the polymer chains and between the nanotube and the polymer chains were modeled with the Lennard-Jones potential by using the parameters used previously. ${ }^{22,27}$ The system was then equilibrated at $300 \mathrm{~K}$ via the MD simulation. The simulations were carried out using DL$\mathrm{POLY}^{\bigcirc},{ }^{28}$ which is a package of molecular simulation subroutines. The systems simulated here are similar to those used in prior work. ${ }^{22}$

Because the equivalent-continuum modeling technique used in this study requires the interaction of the atoms in the molecular model to be described in terms of molecular-mechanics force constants, the interaction of the atoms were defined in terms of bond stretching, bond-angle variation, and Van der Waals interactions. $^{23}$ The force constants used for both models are shown in Tables 1-3, and were taken from the AMBER force field. ${ }^{29}$ A detailed discussion of the parameters used in Tables 1-3 may be found elsewhere. ${ }^{23,29}$ The force constants were assigned with respect to the structure indicated in Fig. 3. The atoms labeled as $\mathrm{CA}, \mathrm{CT}$, and $\mathrm{HC}$ correspond to aromatic carbon atoms ( $\mathrm{sp}^{2}$ orbital), aliphatic carbon atoms $\left(\mathrm{sp}^{3}\right.$ orbital), and hydrogen atoms that are attached to aliphatic carbon atoms, respectively. In the nonfunctionalized system, all of the carbon atoms in the nanotube were modeled as aromatic carbon atoms. 
Truss model

To implement the resultant equivalent-truss structures, finite element models were developed by using ANSYS ${ }^{\circledR} 6^{30}$ (middle portion of Figs. 1 and 2). Each element (LINK8) was a three-dimensional pinjointed truss element with six degrees of freedom (three displacement components on each end) that represented a single atomic interaction. Each node corresponded to an atom in the equilibrium structure of the molecular model. The Young's moduli of the truss elements were determined as described elsewhere. ${ }^{23}$ A total of 36,907 elements and 4751 nodes were used in the model of the nonfunctionalized nanotube, and a total of 36,481 elements and 4747 nodes were used for the model of the functionalized nanotube.

\section{Continuum model}

With the equivalent-truss structure in place, the continuum models were constructed. The geometries of the homogeneous, equivalent-continuum RVEs were assumed to be cylindrical, similar to that of the molecular and truss models (right side of Figs. 1 and 2). The mechanical properties of the continuum solid cylinders were determined by equating the total strain energies of the equivalent-truss and equivalentcontinuum models under identical loading conditions. $^{24,23}$ The molecular model of the functionalized nanotube/polyethylene composite was assumed to exhibit orthotropic symmetry. There are nine independent material parameters required to determine the entire set of elastic constants for an orthotropic material and each of the nine parameters may be determined from a single boundary condition applied to both equivalent-truss and equivalentcontinuum models. The non-functionalized molecular model had transversely-isotropic symmetry, and therefore could be completely described by only five independent parameters. However, for consistency and without loss of accuracy, nine independent parameters were determined with the same nine boundary conditions used in the functionalized model. Once the mechanical properties of the equivalentcontinuum RVEs were determined, the RVEs were assumed to behave in the composite as effective fibers and were used in subsequent micromechanical analyses.

Effective-fiber constitutive model

The constitutive relationship of an orthotropic equivalent-continuum RVE (which is referred to as the effective fiber throughout the remainder of the paper) is

$$
\begin{gathered}
\sigma_{11}=C_{11}^{f} \varepsilon_{11}+C_{12}^{f} \varepsilon_{22}+C_{13}^{f} \varepsilon_{33} \\
\sigma_{22}=C_{12}^{f} \varepsilon_{11}+C_{22}^{f} \varepsilon_{22}+C_{23}^{f} \varepsilon_{33} \\
\sigma_{33}=C_{13}^{f} \varepsilon_{11}+C_{23}^{f} \varepsilon_{22}+C_{33}^{f} \varepsilon_{33} \\
\sigma_{23}=2 C_{44}^{f} \varepsilon_{23} \\
\sigma_{13}=2 C_{55}^{f} \varepsilon_{13} \\
\sigma_{12}=2 C_{66}^{f} \varepsilon_{12}
\end{gathered}
$$

where $\sigma_{i j}$ and $\varepsilon_{i j}$ are the stress and strain components, respectively $(i, j=1,2,3)$, and $C_{k l}^{f}$ are the elastic stiffness components of the effective fiber (denoted by superscript $f$ ) which are written in the usual contracted notation $(k, l=1, . ., 6)$.

Nine independent elastic properties are required to describe the overall elastic behavior of an orthotropic material, as shown in Eq. (1). For convenience, the nine independent elastic properties that can be used to describe the overall behavior of the effective fiber are the three elastic axial stiffness components, $C_{11}^{f}, C_{22}^{f}$, and $C_{33}^{f}$; the three plane-strain bulk moduli, $K_{23}^{f}, K_{13}^{f}$, and $K_{12}^{f}$; and the three elastic shear-stiffness components, $C_{44}^{f}, C_{55}^{f}$, and $C_{66}^{f}$. The three plane-strain bulk moduli are defined as

$$
\begin{aligned}
& K_{23}^{f}=C_{22}^{f}+C_{33}^{f}+2 C_{23}^{f} \\
& K_{13}^{f}=C_{11}^{f}+C_{33}^{f}+2 C_{13}^{f} \\
& K_{12}^{f}=C_{11}^{f}+C_{22}^{f}+2 C_{12}^{f}
\end{aligned}
$$

where the subscripts indicate the plane that is subjected to a plane-strain deformation. Equation (2) can be easily derived in a manner similar to the derivation of the transverse bulk modulus for a transversely-isotropic material. ${ }^{23}$ Once the nine independent elastic properties are determined, the elastic interaction stiffness components can be calculated from the relations in Eq. (2), that is,

$$
\begin{aligned}
& C_{23}^{f}=\frac{1}{2}\left(K_{23}^{f}-C_{22}^{f}-C_{33}^{f}\right) \\
& C_{13}^{f}=\frac{1}{2}\left(K_{13}^{f}-C_{11}^{f}-C_{33}^{f}\right) \\
& C_{12}^{f}=\frac{1}{2}\left(K_{12}^{f}-C_{11}^{f}-C_{22}^{f}\right)
\end{aligned}
$$

At this point, the values of the nine elastic parameters are unknown. These values are determined by applying nine identical sets of boundary conditions to the equivalent-truss model and the effective fiber, and by 
subsequently equating the strain energies by adjusting the nine independent elastic properties. The boundary conditions used were chosen such that each of the nine corresponding strain energies of the effective fiber was dependent on only one parameter.

Boundary conditions

Nine sets of boundary conditions were chosen to uniquely determine each of the nine independent elastic properties. The displacements applied at the boundaries of the RVE are generalized by

$$
u_{i}(B)=\varepsilon_{i j} x_{j}
$$

where $B$ is the bounding surface, $x_{j}$ is defined in Figs. 1 and 2 , and the summation convention associated with repeated indices is used. The total strain energy of the effective fiber is

$$
\Lambda^{f}=\frac{V}{2} \sigma_{i j} \varepsilon_{i j}
$$

where $V$ is the volume of the effective fiber and includes the boundary region surrounding the nanotube. The boundary conditions and strain energies for each of the nine independent elastic properties are listed in Tables 4-6. Unspecified strain components in Tables 4-6 are zero valued.

Boundary region

The displacements specified in Tables 4-6 were applied to each node in the boundary region of the equivalent-truss model (indicated in Figs. 1 and 2), and the corresponding strain energies were calculated by summing the strain energies of each individual truss member in the RVE.

It was assumed that the thickness of the boundary region was equal to the maximum distance for which a positive-definite relationship exists between the force and displacement of all of the atom types. It was also assumed that the contribution of the energies associated with van der Waals forces between atoms with a larger distance than this maximum were relatively small and could be neglected.

Based on the repeating unit used in the MD simulation, the effective fiber radius and length were chosen to be $1.1 \mathrm{~nm} 4.3 \mathrm{~nm}$, respectively. The calculated values of the nine independent parameters for the functionalized and non-functionalized effective fibers are listed in Table 7.

\section{Micromechanical modeling}

Constitutive models of the effective fiber/polymer composite were developed with a micromechanical analysis by using the mechanical properties of the effective fiber and the bulk polymer matrix material. For the composites considered in this study, the polymer molecules that were near the polymer/nanotube interface were included in the effective fiber, and it was assumed that the matrix polymer surrounding the effective fiber had mechanical properties equal to those of bulk polyethylene. All relative movement and interaction of the polymer chains with respect to each other was modeled at the molecular level. This motion and interaction is therefore indirectly considered in the subsequent determination of the properties for the effective fibers, and it is therefore assumed that perfect bonding exists between the effective fiber and the surrounding polymer matrix in the micromechanics analysis.

The micromechanics-based Mori-Tanaka method $^{31,32}$ was used to predict the elastic mechanical properties of the composite material considered herein. For this method, the overall elastic-stiffness tensor of the composite containing orthotropic effective fibers embedded in a matrix material is

$$
\mathbf{C}=\left(c_{m} \mathbf{C}^{m}+c_{f}\left\langle\mathbf{C}^{f} \mathbf{T}^{f}\right\rangle\right)\left(c_{m} \mathbf{I}+c_{f}\left\langle\mathbf{T}^{f}\right\rangle\right)^{-1}
$$

where $c_{f}$ and $c_{m}$ are the effective fiber and matrix volume fractions, respectively, $\mathbf{C}^{f}$ and $\mathbf{C}^{m}$ are the stiffness tensors of the effective fiber and matrix, respectively, $\mathbf{I}$ is the identity tensor, the angle-brackets indicate an effective-fiber orientation average, and $\mathbf{T}^{f}$ is the dilute strain-concentration tensor of the effective fibers, and is given by

$$
\mathbf{T}^{f}=\left[\mathbf{I}+\mathbf{S}^{f}\left(\mathbf{C}^{m}\right)^{-1}\left(\mathbf{C}^{f}-\mathbf{C}^{m}\right)\right]^{-1}
$$

where $\mathbf{S}^{f}$ is the Eshelby tensor. ${ }^{33}$ For unidirectionallyaligned effective fibers, the orientation averaging in Eq. (6) is not necessary, and the resulting elastic stiffness components of the composite have orthotropic symmetry. For three-dimensional randomly oriented effective fibers, the orientation average of a tensor, $\mathbf{A}$, is

$$
\begin{aligned}
\langle\mathbf{A}\rangle=\left\langle A_{i j m n}\right\rangle= & \left(\kappa-\frac{2}{3} \mu\right)\left(\delta_{i j} \delta_{m n}\right) \\
& +\mu\left(\delta_{i s} \delta_{j n}+\delta_{i n} \delta_{j m}\right)
\end{aligned}
$$

where $i, j, m, n=1,2,3$; the indicial summation convention is used; $\delta_{i j}$ is the Kronecker delta; and 


$$
\begin{gathered}
\kappa=\frac{1}{9} A_{i i j j} \\
\mu=\frac{1}{10}\left(A_{i j i j}-\frac{1}{3} A_{i i j j}\right)
\end{gathered}
$$

Therefore, from Eqs. (8) and (9), $\langle\mathbf{A}\rangle$ is isotropic.

For the effective fiber/polymer composite considered in the present study, the elastic stiffness components, volume fraction, length, and orientation of the effective fiber were used for the inclusion properties in Eq. (6). The effective fibers were assumed to have an spheroidal geometry for the Eshelby tensor, so that any length of effective fiber could be modeled. The effective-fiber length was adjusted by varying the inclusion aspect ratio while holding the diameter constant. While the nanotube and effective-fiber lengths are equivalent, the nanotube volume fraction was determined to be $52.9 \%$ of the effective-fiber volume fraction. This value was calculated by assuming the nanotube volume is defined as the total space occupied by the nanotube, including half of the unoccupied space between the nanotube and polymer. It was assumed that the polyethylene matrix was isotropic, with a representative Young's modulus and Poisson's ratio of $0.9 \mathrm{GPa}$ and 0.3 , respectively. The overall composite stiffness was calculated for various effective-fiber lengths and volume fractions for both cases of perfectly aligned and three-dimensional randomlyoriented effective fibers.

\section{$\underline{\text { Results }}$}

In this section, the moduli of the nanotube composites are presented in terms of nanotube length, volume fraction, and orientation. While the shear moduli of the composite material $\left(\mathrm{G}_{44}, \mathrm{G}_{55}, \mathrm{G}_{66}\right)$ are simply equal to the shear-stiffness components $\left(\mathrm{C}_{44}\right.$, $\left.\mathrm{C}_{55}, \mathrm{C}_{66}\right)$, the Young's moduli $\left(\mathrm{E}_{1}, \mathrm{E}_{2}, \mathrm{E}_{3}\right)$ were calculated by using the components of the compliance tensor of the composite material, which was determined by inverting the composite stiffness tensor, C. ${ }^{34}$ The subscripts of the Young's moduli and shear moduli indicate the principal direction associated with the quantity, similar to Eq. (1). Therefore, $E_{1}$ is the longitudinal Young's modulus (parallel to the nanotube), $\mathrm{E}_{2}$ and $\mathrm{E}_{3}$ are transverse Young's moduli, $\mathrm{G}_{44}$ is the transverse shear modulus, and $\mathrm{G}_{55}$ and $\mathrm{G}_{66}$ are longitudinal shear moduli. Since the random composites are isotropic, their mechanical properties are completely described by their Young's modulus, E $\left(\mathrm{E}=\mathrm{E}_{1}=\mathrm{E}_{2}=\mathrm{E}_{3}\right)$, and shear modulus, $\mathrm{G}\left(\mathrm{G}=\mathrm{G}_{44}=\right.$ $\left.\mathrm{G}_{55}=\mathrm{G}_{66}\right)$.
The Young's modulus of the nanotube composite is shown in Fig. 4 as a function of nanotube length, for a $1 \%$ nanotube volume fraction. The longitudinal Young's modulus of the aligned composite and the Young's modulus of the random composite have the expected non-linear dependence on the nanotube length. An increase in the Young's modulus with respect to an increase in nanotube length is expected to correspond to a relative increase in load transfer efficiency between the nanotube and the surrounding polymer. The data in Fig. 4 indicate that at a nanotube length of about $400 \mathrm{~nm}$, the efficiency of load transfer is nearly maximized. Further increases in nanotube length result in relatively small increases in Young's modulus for a given volume fraction. As the nanotube length becomes greater than approximately $100 \mathrm{~nm}$, the Young's modulus for the non-functionalized composite becomes larger than that of the functionalized composite for the random composite and longitudinal deformation of the aligned composite. By contrast, the transverse Young's modulus of the aligned composite has no dependence on nanotube length. Also, there is no effect of functionalization on the transverse deformation of the aligned composite.

The shear modulus of the nanotube composite is shown in Fig. 5 as a function of nanotube length. The shear modulus of the random composite shows an expected non-linear dependence on nanotube length, whereas the shear modulus of the aligned composite has no dependence on nanotube-length. Again, the data indicate that the efficiency of load transfer, as evidenced by a modulus change, is nearly maximized at a nanotube length of about $400 \mathrm{~nm}$. For the random composite, the shear modulus of the non-functionalized system increases over that of the functionalized system as the nanotube length is increased. There is no significant effect of functionalization on the shear modulus of the aligned composite as the nanotube length is increased. As the nanotube length approaches the length of the effective fiber, $4.3 \mathrm{~nm}$, the shear modulus is significantly higher than the shear modulus of the polyethylene matrix, $0.35 \mathrm{GPa}$ (calculated using the isotropic polyethylene properties given in the previous section), which indicates the large effect of small inclusions on the shear modulus at fixed concentrations.

The Young's moduli of the random and aligned composites are shown in Figs. 6 and 7 as a function of nanotube volume fraction, for a constant nanotube length of $400 \mathrm{~nm}$. At this nanotube length, a change in the longitudinal Young's modulus with respect to volume fraction of the aligned composite is nearly linear, resembling a linear rule-of-mixtures relationship. Over the complete range of volume fraction, Young's modulus for the non-functionalized composite is greater than that of the functionalized composite for both the 
random composite and longitudinal deformation of the aligned composite. Conversely, for the transverse deformation of the aligned composite, the Young's modulus of the aligned, functionalized composite is greater than the non-functionalized material.

The shear modulus of the nanotube composite is shown in Fig. 8 as a function of nanotube volume fraction. As the nanotube volume fraction increases, the shear modulus for the non-functionalized composite becomes greater than that of the functionalized composite for the random-nanotube orientation. There is no significant effect of the functionalization on the shear modulus of the aligned composite for the given range of nanotube volume fractions.

\section{Summary and Conclusions}

In this study, the bulk composite elastic properties of a nanotube/polyethylene composite have been predicted for both functionalized and non-functionalized nanotubes. It was assumed that functionalization would effect the load transfer between the nanotube and the surrounding matrix, and that changes in predicted elastic stiffness would be directly related to the efficiency of the load-transfer mechanism. The analysis method establishes constitutive equations for both functionalized and non-functionalized nanotube composites systems by using a hierarchical equivalent-continuum modeling technique that predicts elastic bulk behavior, using intrinsic properties developed through molecular dynamics simulations. The elastic properties of the functionalized and non-functionalized composites systems have been examined for various nanotube lengths, volume fractions, and both random and aligned nanotube orientations.

For a fixed nanotube volume fraction of $1 \%$ and various nanotube lengths, the Young's modulus of the random composite, the shear modulus of the random composite, and the longitudinal Young's modulus of the aligned composite have been shown to decrease up to $10 \%$ when the nanotube is functionalized. For a fixed nanotube length of $400 \mathrm{~nm}$ and various nanotube volume fractions, the longitudinal Young's modulus of the aligned composite, the Young's modulus of the random composite, and the shear modulus of the random composite have also been shown to decrease up to $12 \%$ when the nanotube is functionalized. However, under these conditions, the transverse Young's modulus of the aligned composite has shown an increase of up to $24 \%$ when the nanotube is functionalized.

The primary implication of these results is that although chemical functionalization of carbon nanotubes has been considered a possible means to increase load-transfer efficiency in a nanotube/polymer composite, this functionalization has in fact degraded the macroscopic elastic stiffness of the composite considered in this study. In general, occurs for both random composites and aligned composites over a wide range of nanotube volume fractions and lengths.

\section{Acknowledgements}

This research was partially supported by the National Aeronautics and Space Administration under NASA contract No. NAS1-97046 while Dr. Odegard and Dr. Frankland were in residence at ICASE, NASA Langley Research Center, Hampton, VA 23681-2199.

\section{References}

1) Thostenson, E.T.; Ren, Z., and Chou, T.W., "Advances in the Science and Technology of Carbon Nanotubes and Their Composites: A Review," Composites Science and Technology, Vol. 61, 2001, pp. 1899-1912.

2) Sun, Y.P.; Fu, K.; Lin, Y., and Huang, W., "Functionalized Carbon Nanotubes: Properties and Applications," Accounts of Chemical Research, Vol. 35, 2002, pp. 1096-1104.

3) Chen, J.; Hamon, M.A.; Hu, H.; Chen, Y.; Rao, A.M.; Eklund, P.C., and Haddon, R.C., "Solutions Properties of Single-Walled Carbon Nanotubes," Science, Vol. 282, 1998, pp. 95-98.

4) Bahr, J.L. and Tour, J.M., "Highly Functionalized Carbon Nanotubes Using In Situ Generated Diazonium Compounds," Chemistry of Materials, Vol. 13, 2001, pp. 3823-3824.

5) Michelson, E.T.; Huffman, C.B.; Rinzler, A.G.; Smalley, R.E.; Hauge, R.H., and Margrave, J.L., "Fluorination of Buckytubes," Chemical Physics Letters, Vol. 296, 1998, pp. 188-194.

6) Michelson, E.T.; Chiang, I.W.; Zimmerman, J.L.; Boul, P.J.; Lozano, J.; Liu, J.; Smalley, R.E.; Hauge, R.H., and Margrave, J.L., "Solvation of Fluorinated Single-Wall Carbon Nanotubes in Alcohol Solvents," Journal of Physical Chemistry B, Vol. 103, 1999, pp. 4318-4322.

7) Boul, P.J.; Liu, J.; Michelson, E.T.; Huffman, C.B.; Ericson, L.M.; Chiang, I.W.; Smith, K.A.; Colbert, D.T.; Hauge, R.H.; Margrave, J.L., and Smalley, R.E., "Reversible Sidewall Functionalization of Buckytubes," Chemical Physics Letters, Vol. 310, 1999, pp. 367-372.

8) Chen, Y.; Haddon, R.C.; Fang, S.; Rao, A.M.; Eklund, P.C.; Lee, W.H.; Dickey, E.C.; Grulke, E.A.; Pendergrass, J.C.; Chavan, A.; Haley, B.E., and Smalley, R.E., "Chemical Attachment of Organic Functional Groups to Single-Walled Carbon Nanotube Material," Journal of Materials Research, Vol. 13, No. 9, 1998, pp. 2423-2431. 
9) Jin, Z.; Sun, X.; Xu, G.; Goh, S.H., and Ji, W., "Non-Linear Optical Properties of Some

Polymer/Multi-Walled Carbon Nanotube Composites," Chemical Physics Letters, Vol. 318, 2000, pp. 505-510.

10) Hill, D.E.; Lin, Y.; Rao, A.M.; Allard, L.F., and Sun, Y.P., "Functionalization of Carbon Nanotubes with Polystyrene," Macromolecules, Vol. 35, 2002, pp. 9466-9471.

11) Lin, Y.; Rao, A.M.; Sadanadan, B.; Kenik, E.A., and Sun, Y.P., "Functionalizing Multiple-Walled Carbon Nanotubes with Aminopolymers," Journal of

Physical Chemistry B, Vol. 106, 2002, pp. 1294-1298.

12) Huang, W.; Taylor, S.; Fu, K.; Lin, Y.; Zhang, D.; Hanks, T.W.; Rao, A.M., and Sun, Y.P.,

"Attaching Proteins to Carbon Nanotubes via DiimideActivated Amidation," NanoLetters, Vol. 2, 2002, pp. 311-314.

13) Velasco-Santos, C.; Martinez-Hernandez, A.L.; Lozada-Cassou, M.; Alvarex-Castillo, A., and Castano, V.M., "Chemical Functionalization of Carbon Nanotubes Through an Organosilane," Nanotechnology, Vol. 13, 2002, pp. 495-498.

14) Banerjee, S. and Wong, S.S., "Structural Characterization, Optical Properties, and Improved Solubility of Carbon Nanotubes Functionalized with Wilkinson's Catalyst," Journal of the American Chemical Society, Vol. 124, 2002, pp. 8940-8948.

15) Chen, R.J.; Zhang, Y.; Wang, D., and Dai, H., "Noncovalent Sidewall Functionalization of SingleWalled Carbon Nanotubes for Protein Immobilization," Journal of the American Chemical Society, Vol. 123, 2001, pp. 3838-3839.

16) Star, A.; Stoddart, J.F.; Steuerman, D.; Diehl, M.; Boukai, A.; Wong, E.W.; Yang, X.; Chung, S.; Choi, H., and Heath, J.R., "Preparation and Properties of Polymer-Wrapped Single-Walled Carbon Nanotubes," Angewandte Chemie International Edition in English, Vol. 40, No. 9, 2001, pp. 17211725.

17) Lordi, V. and Yao, N., "Molecular Mechanics of Binding in Carbon-Nanotube-Polymer Composites," Journal of Materials Research, Vol. 15, 2000, pp. 2770-2779.

18) Jia, Z.; Wang, Z.; Xu, C.; Liang, J.; Wei, B.; Wu, D., and Zhu, S., "Study on Poly(Methyl Methacrylate)/Carbon Nanotube Composites," Materials Science and Engineering A, Vol. A271, 1999, pp. 395-400.

19) Wagner, H.D.; Lourie, O.; Feldman, Y., and Tenne, R., "Stress-Induced Fragmentation of Multiwall Carbon Nanotubes in a Polymer Matrix," Applied Physics Letters, Vol. 72, 1998, pp. 188-190.

20) Georgakilas, V.; Kordatos, K.; Prato, M.; Guldi, D.M.; Holzinger, M., and Hirsch, A., "Organic Functionalization of Carbon Nanotubes," Journal of the American Chemical Society, Vol. 124, 2002, pp. 760-761.

21) Georgakilas, V.; Voulgaris, D.; Vazquez, E.; Prato, M.; Guldi, D.M.; Kukovecz, A., and Kuzmany, H., "Purification of HiPCO Carbon Nanotubes via Organic Functionalization," Journal of the American Chemical Society, Vol. 124, 2002, pp. 14318-14319.

22) Frankland, S.J.V.; Caglar, A.; Brenner, D.W., and Griebel, M., "Molecular Simulation of the Influence of Chemical Cross-Links on the Shear Strength of Carbon nanotube-Polymer Interfaces," Journal of Physical Chemistry B, Vol. 106, 2002, pp. 3046-3048.

23) Odegard, G.M.; Gates, T.S.; Wise, K.E.; Park, C., and Siochi, E.J., "Constitutive Modeling of NanotubeReinforced Polymer Composites," NASA/CR-2002$211760,2002$.

24) Odegard, G.M.; Gates, T.S.; Nicholson, L.M., and Wise, K.E., "Equivalent-Continuum Modeling of NanoStructured Materials," Composites Science and Technology, Vol. 62, No. 14, 2002, pp. 1869-1880.

25) Rappe, A.K. and Casewit, C.J., Molecular Mechanics Across Chemistry, University Science Books, Sausalito, California, 1997.

26) Brenner, D.W.; Shenderova, O.A.; Harrison, J.A.; Stuart, S.J.; Ni, B., and Sinnott, S.B., "Second Generation Reactive Empirical Bond Order (REBO) Potential Energy Expression for Hydrocarbons," Journal of Physics C: Condensed Matter, Vol. 14, 2002, pp. 783.

27) Allen, M.P. and Tildesley, D.J., Computer Simulation of Liquids, Oxford University Press, Oxford, 1987.

28) Smith, W. and Forester, T.R., DL-POLY, Daresbury, Nr. Warrington, England, The Council for the Central Laboratory of the Research Councils, 1996.

29) Cornell, W.D.; Cieplak, P.; Bayly, C.I.; Gould, I.R.; Merz, K.M.; Ferguson, D.M.; Spellmeyer, D.C.; Fox, T.; Caldwell, J.W., and Kollman, P.A., "A Second Generation Force Field for the Simulation of Proteins, Nucleic Acids, and Organic Molecules," Journal of the American Chemical Society, Vol. 117, 1995, pp. 51795197.

30) ANSYS, Ver. 6, Canonsburg, PA, SAS IP, 2001.

31) Mori, T. and Tanaka, K., "Average Stress in Matrix and Average Elastic Energy of Materials with Misfitting Inclusions," Acta Metallurgica, Vol. 21, 1973, pp. 571-574.

32) Benveniste, Y., "A New Approach to the Application of Mori-Tanaka's Theory in Composite Materials," Mechanics of Materials, Vol. 6, 1987, pp. 147-157.

33) Eshelby, J.D., "The Determination of the Elastic Field of an Ellipsoidal Inclusion, and Related Problems," Proceedings of the Royal Society of London, Series A, Vol. A241, 1957, pp. 376-396. 
34) Hull, D. and Clyne, T.W., An Introduction to

Composite Materials, Cambridge University Press,

Cambridge, 1996.

Table 1. Bond-stretching parameters

\begin{tabular}{|c|c|c|}
\hline $\begin{array}{c}\text { Bond } \\
\text { stretching }\end{array}$ & $\mathrm{P}(\AA)$ & $\begin{array}{c}K^{\mathrm{P}} \\
\left(\mathrm{kcal} / \mathrm{mole} / \AA^{2}\right)\end{array}$ \\
\hline CA-CA & 1.400 & 469 \\
\hline CA-CT & 1.510 & 317 \\
\hline CT-CT & 1.526 & 310 \\
\hline CT-HC & 1.090 & 340 \\
\hline
\end{tabular}

Table 2. Bond-angle variation parameters

\begin{tabular}{|c|c|c|}
\hline $\begin{array}{c}\text { Bond-angle } \\
\text { variation }\end{array}$ & $\Theta(\mathrm{deg})$ & $\begin{array}{c}K^{\ominus} \\
\left(\mathrm{kcal} / \mathrm{mole} / \mathrm{rad}^{2}\right)\end{array}$ \\
\hline CA-CA-CA & 120.0 & 63 \\
\hline CA-CA-CT & 120.0 & 70 \\
\hline CA-CT-CA & 120.0 & 63 \\
\hline CA-CT-CT & 114.0 & 63 \\
\hline CT-CT-CT & 109.5 & 40 \\
\hline CT-CT-HC & 109.5 & 50 \\
\hline HC-CT-HC & 109.5 & 35 \\
\hline
\end{tabular}

Table 3. Van der Waals interaction parameters

\begin{tabular}{|c|c|c|}
\hline $\begin{array}{c}\text { Van der Waals } \\
\text { interaction }\end{array}$ & $\begin{array}{c}D^{\mathrm{I}} \\
(\mathrm{kcal} / \mathrm{mole})\end{array}$ & $\rho^{\mathrm{I}}(\AA)$ \\
\hline $\mathrm{CA}$ & 0.0860 & 3.816 \\
\hline $\mathrm{CT}$ & 0.1094 & 3.816 \\
\hline $\mathrm{HC}$ & 0.0157 & 2.974 \\
\hline
\end{tabular}


Table 4. Boundary conditions for axial stiffness components

\begin{tabular}{|c|c|c|c|}
\hline Property & $\begin{array}{l}\text { Boundary } \\
\text { conditions }\end{array}$ & $\begin{array}{c}\text { Boundary } \\
\text { displacements }\end{array}$ & Strain energy \\
\hline$C_{11}^{f}$ & $\varepsilon_{11}=e$ & $\begin{array}{l}u_{1}(B)=e x_{1} \\
u_{2}(B)=0 \\
u_{3}(B)=0\end{array}$ & $\Lambda^{f}=\frac{V}{2} C_{11}^{f} e^{2}$ \\
\hline$C_{22}^{f}$ & $\varepsilon_{22}=e$ & $\begin{array}{l}u_{1}(B)=0 \\
u_{2}(B)=e x_{2} \\
u_{3}(B)=0\end{array}$ & $\Lambda^{f}=\frac{V}{2} C_{22}^{f} e^{2}$ \\
\hline$C_{33}^{f}$ & $\varepsilon_{33}=e$ & $\begin{array}{l}u_{1}(B)=0 \\
u_{2}(B)=0 \\
u_{3}(B)=e x_{3}\end{array}$ & $\Lambda^{f}=\frac{V}{2} C_{33}^{f} e^{2}$ \\
\hline
\end{tabular}

Table 5. Boundary conditions for plane-strain bulk moduli

\begin{tabular}{|c|c|c|c|}
\hline Property & $\begin{array}{c}\text { Boundary } \\
\text { conditions }\end{array}$ & $\begin{array}{c}\text { Boundary } \\
\text { displacements }\end{array}$ & Strain energy \\
\hline$K_{23}^{f}$ & $\varepsilon_{22}=\varepsilon_{33}=e$ & $\begin{array}{l}u_{1}(B)=0 \\
u_{2}(B)=e x_{2} \\
u_{3}(B)=e x_{3}\end{array}$ & $\Lambda^{f}=\frac{V}{2} K_{23}^{f} e^{2}$ \\
\hline$K_{13}^{f}$ & $\varepsilon_{11}=\varepsilon_{33}=e$ & $\begin{array}{l}u_{1}(B)=e x_{1} \\
u_{2}(B)=0 \\
u_{3}(B)=e x_{3}\end{array}$ & $\Lambda^{f}=\frac{V}{2} K_{13}^{f} e^{2}$ \\
\hline$K_{12}^{f}$ & $\varepsilon_{11}=\varepsilon_{22}=e$ & $\begin{array}{l}u_{1}(B)=e x_{1} \\
u_{2}(B)=e x_{2} \\
u_{3}(B)=0\end{array}$ & $\Lambda^{f}=\frac{V}{2} K_{12}^{f} e^{2}$ \\
\hline
\end{tabular}


Table 6. Boundary conditions for shear-stiffness components

\begin{tabular}{|c|c|c|c|}
\hline Property & $\begin{array}{c}\text { Boundary } \\
\text { conditions }\end{array}$ & $\begin{array}{c}\text { Boundary } \\
\text { displacements }\end{array}$ & Strain energy \\
\hline$C_{44}^{f}$ & $\varepsilon_{23}=\gamma / 2$ & $u_{1}(B)=0$ & \\
& $u_{2}(B)=\frac{\gamma}{2} x_{3}$ & $\Lambda^{f}=\frac{V}{2} C_{44}^{f} \gamma^{2}$ \\
& $u_{3}(B)=\frac{\gamma}{2} x_{2}$ & \\
\hline$C_{55}^{f}$ & $\varepsilon_{13}=\gamma / 2$ & $u_{2}(B)=\frac{\gamma}{2} x_{3}$ & \\
& & $u_{3}(B)=\frac{\gamma}{2} x_{1}$ & \\
\hline & & $u_{1}(B)=\frac{\gamma}{2} x_{2}$ & \\
$C_{66}^{f}$ & $\varepsilon_{12}=\gamma / 2$ & $u_{2}(B)=\frac{\gamma}{2} C_{15}^{f} \gamma^{2}$ & $\Lambda^{f}=\frac{V}{2} C_{66}^{f} \gamma^{2}$ \\
& & $u_{3}(B)=0$ & \\
\hline
\end{tabular}

Table 7. Effective-Fiber Properties (GPa)

\begin{tabular}{|c|c|c|}
\hline & $\begin{array}{c}\text { Non-functionalized } \\
\text { nanotube }\end{array}$ & $\begin{array}{c}\text { Functionalized } \\
\text { nanotube }\end{array}$ \\
\hline$C_{11}^{f}$ & 548.7 & 487.7 \\
\hline$C_{22}^{f}$ & 16.8 & 24.5 \\
\hline$C_{33}^{f}$ & 16.5 & 20.6 \\
\hline$K_{23}^{f}$ & 59.0 & 77.9 \\
\hline$K_{13}^{f}$ & 597.1 & 548.2 \\
\hline$K_{12}^{f}$ & 597.0 & 554.6 \\
\hline$C_{44}^{f}$ & 7.1 & 12.7 \\
\hline$C_{55}^{f}$ & 144.0 & 155.4 \\
\hline$C_{66}^{f}$ & 144.9 & 137.0 \\
\hline
\end{tabular}



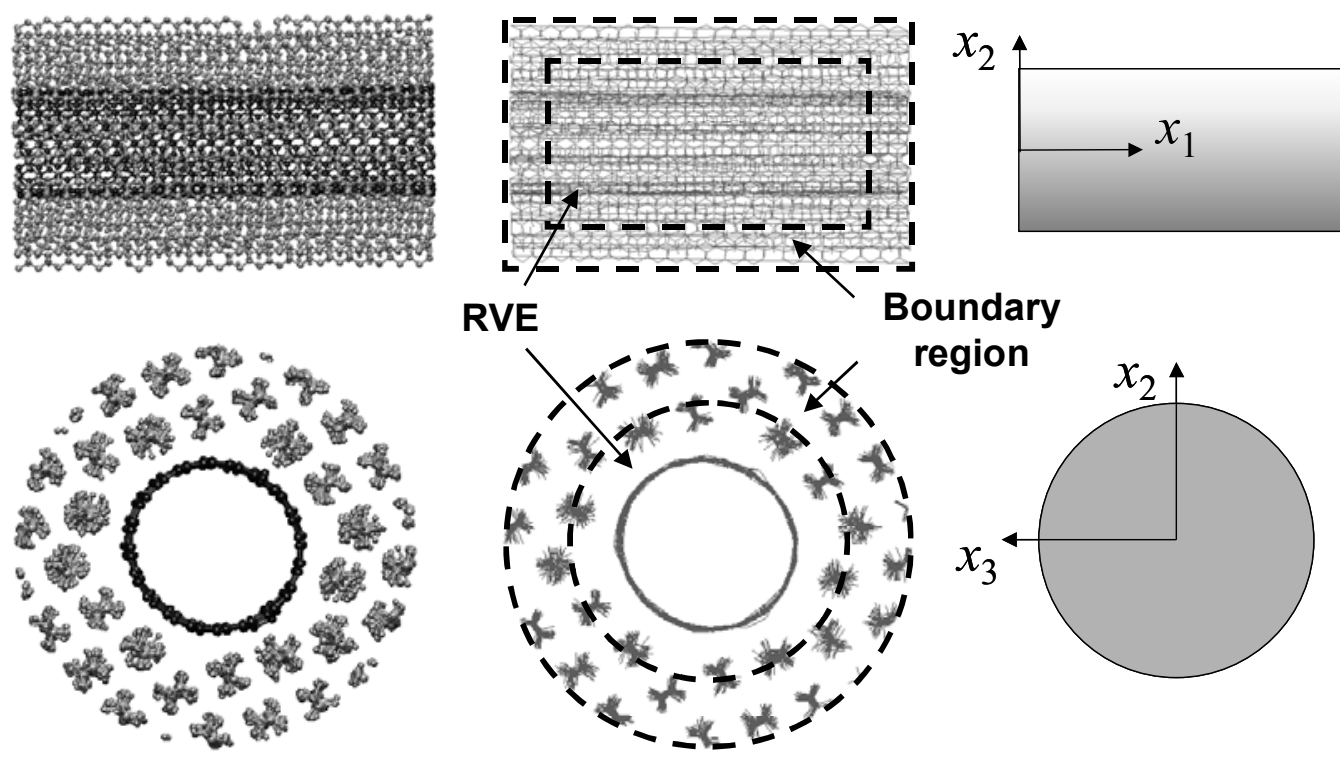

RVE

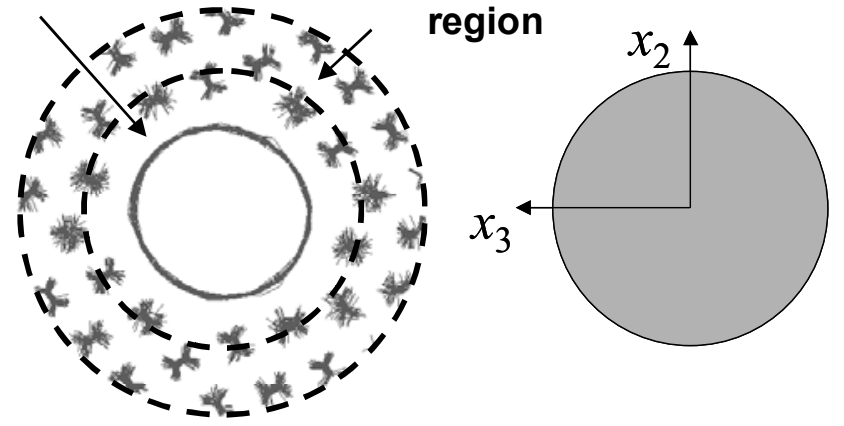

\section{Molecular model}

Truss model

(only bond-stretch elements shown)

\section{Boundary}

Continuum

model

Figure 1. Equivalent-continuum modeling of non-functionalized system
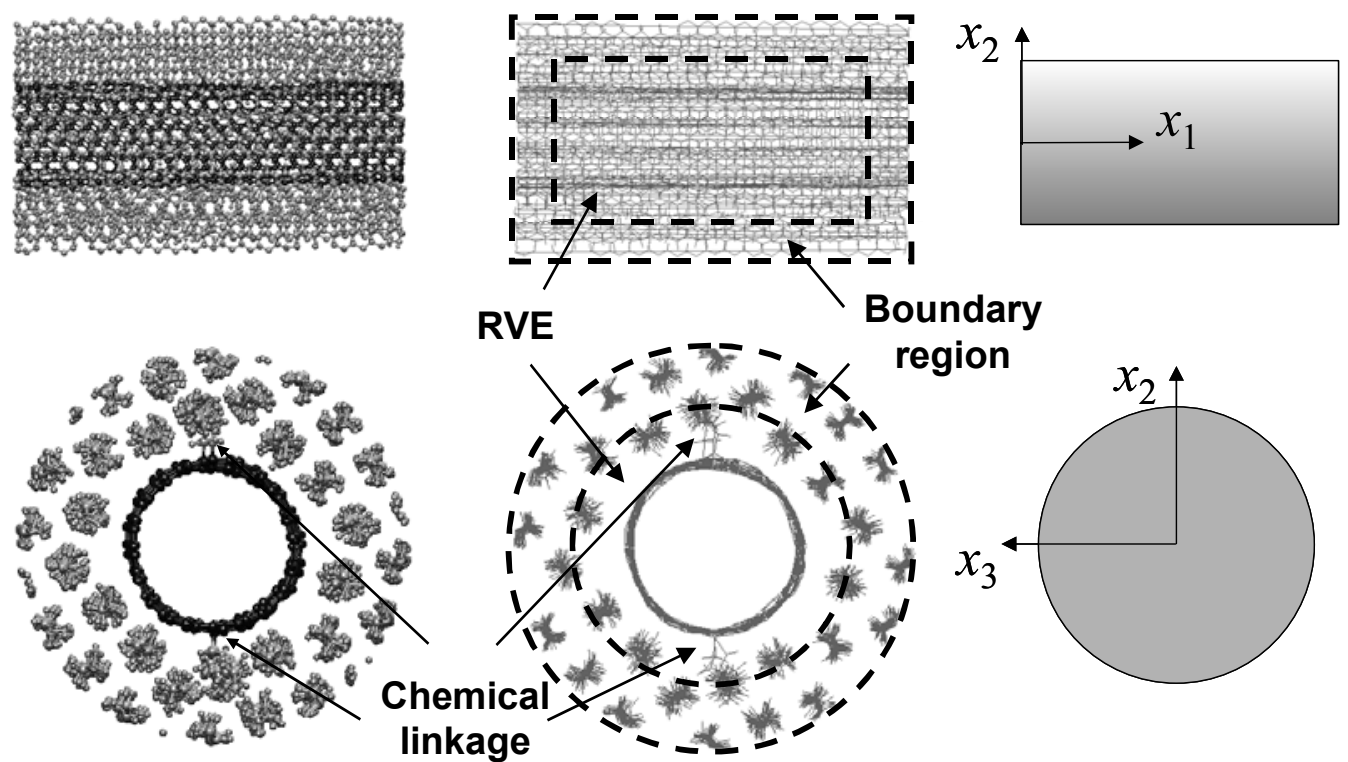

RVE
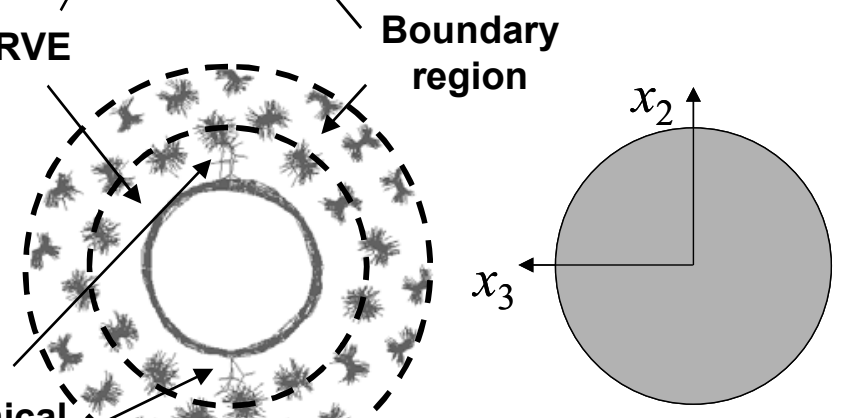

Molecular

Truss model model

(only bond-stretch elements shown)

Continuum

model

Figure 2. Equivalent-continuum modeling of functionalized system 


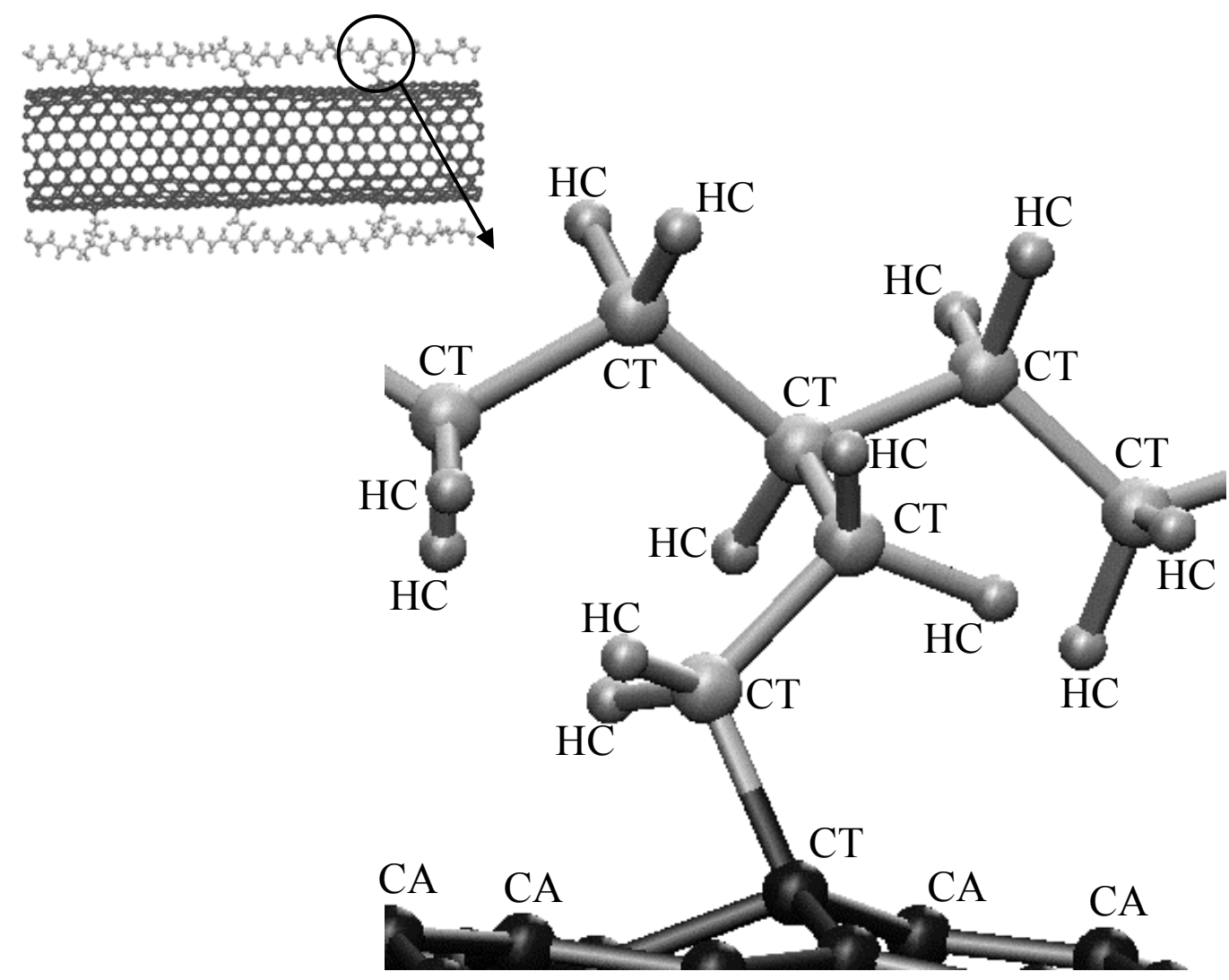

Figure 3. Modeled atomic structure of the functionalized nanotube/polyethylene composite

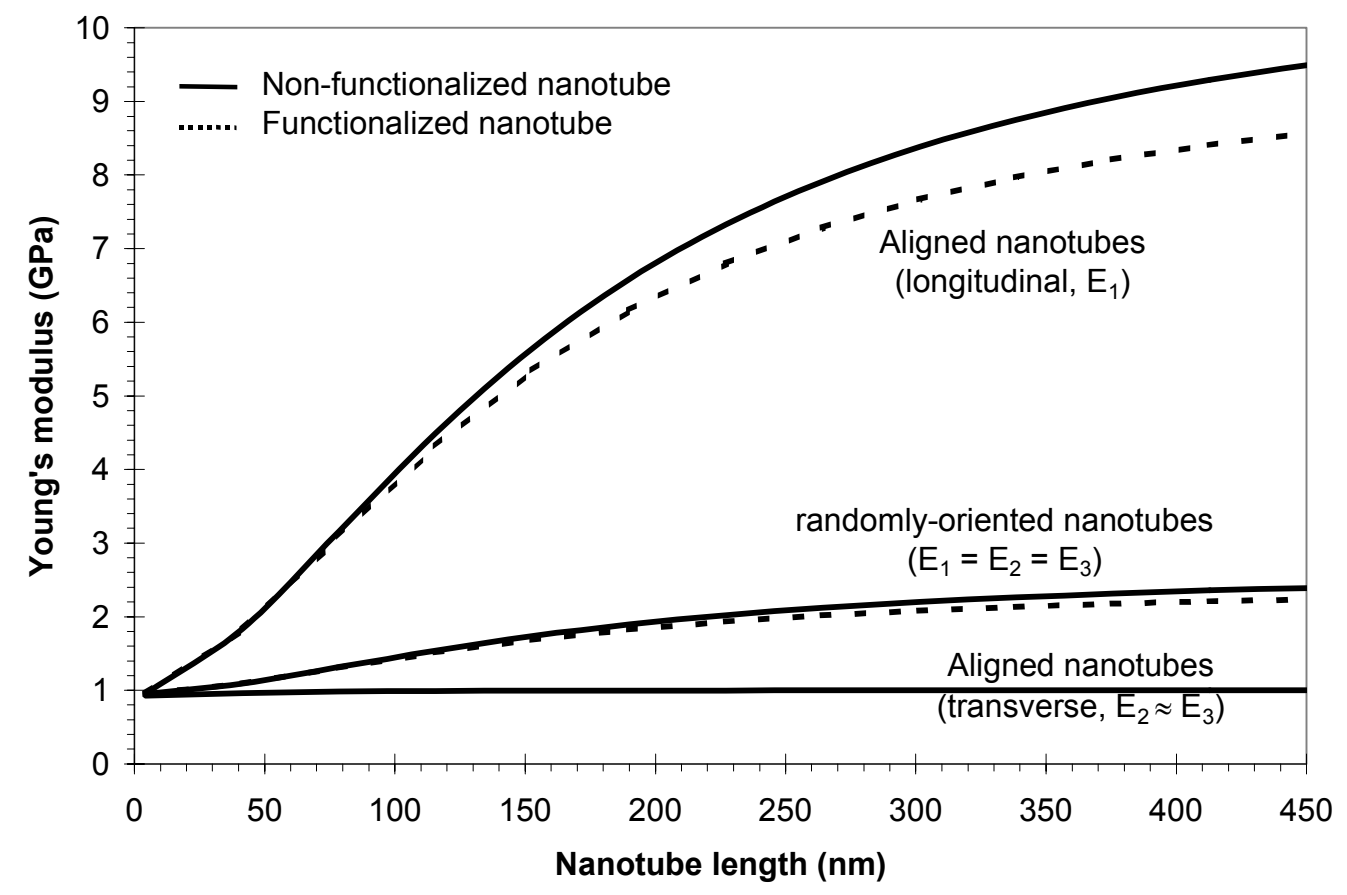

Figure 4. Young's moduli of both composite systems vs. nanotube length for a $1 \%$ nanotube volume fraction 


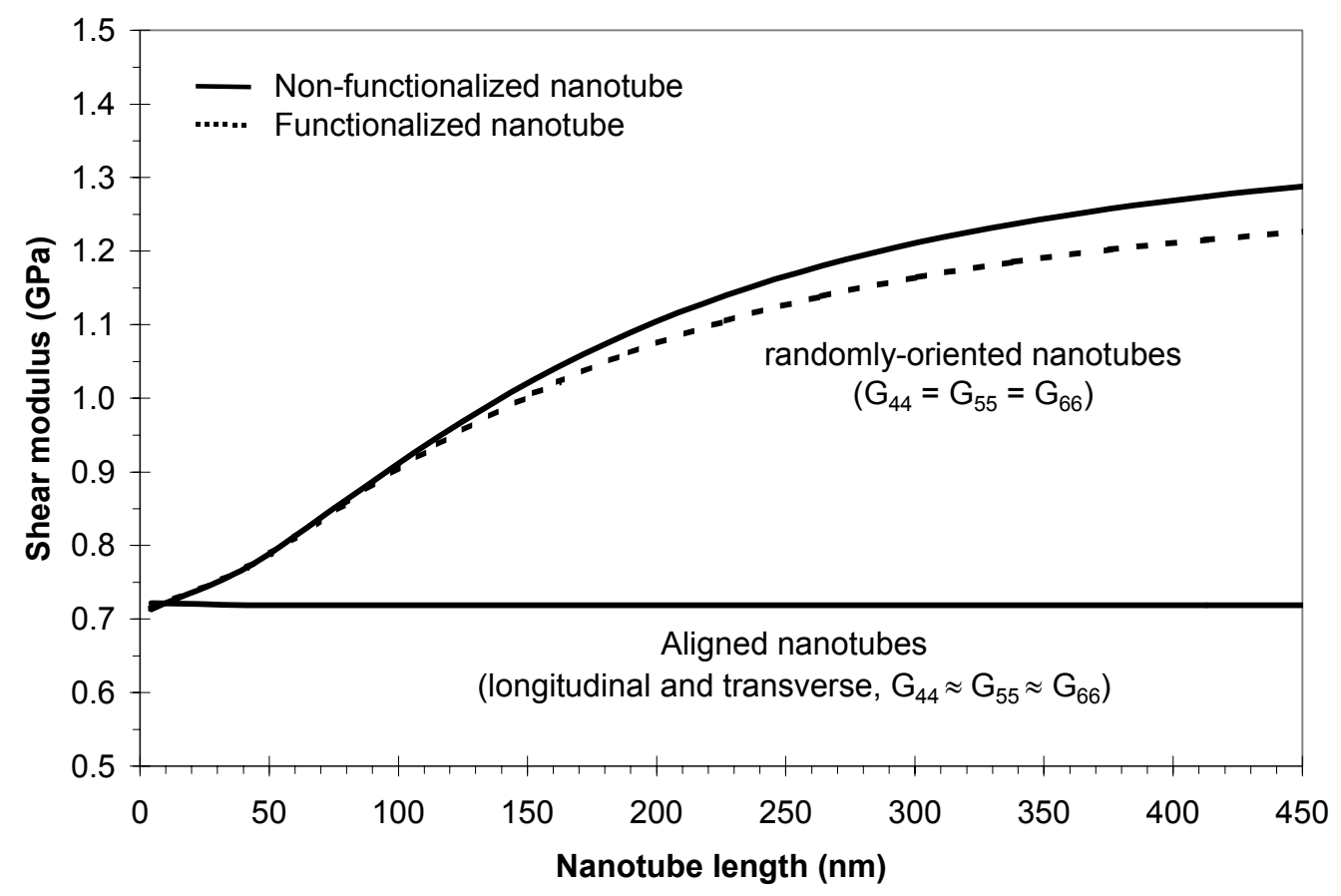

Figure 5. Shear moduli of both composite systems vs. nanotube length for a $1 \%$ nanotube volume fraction

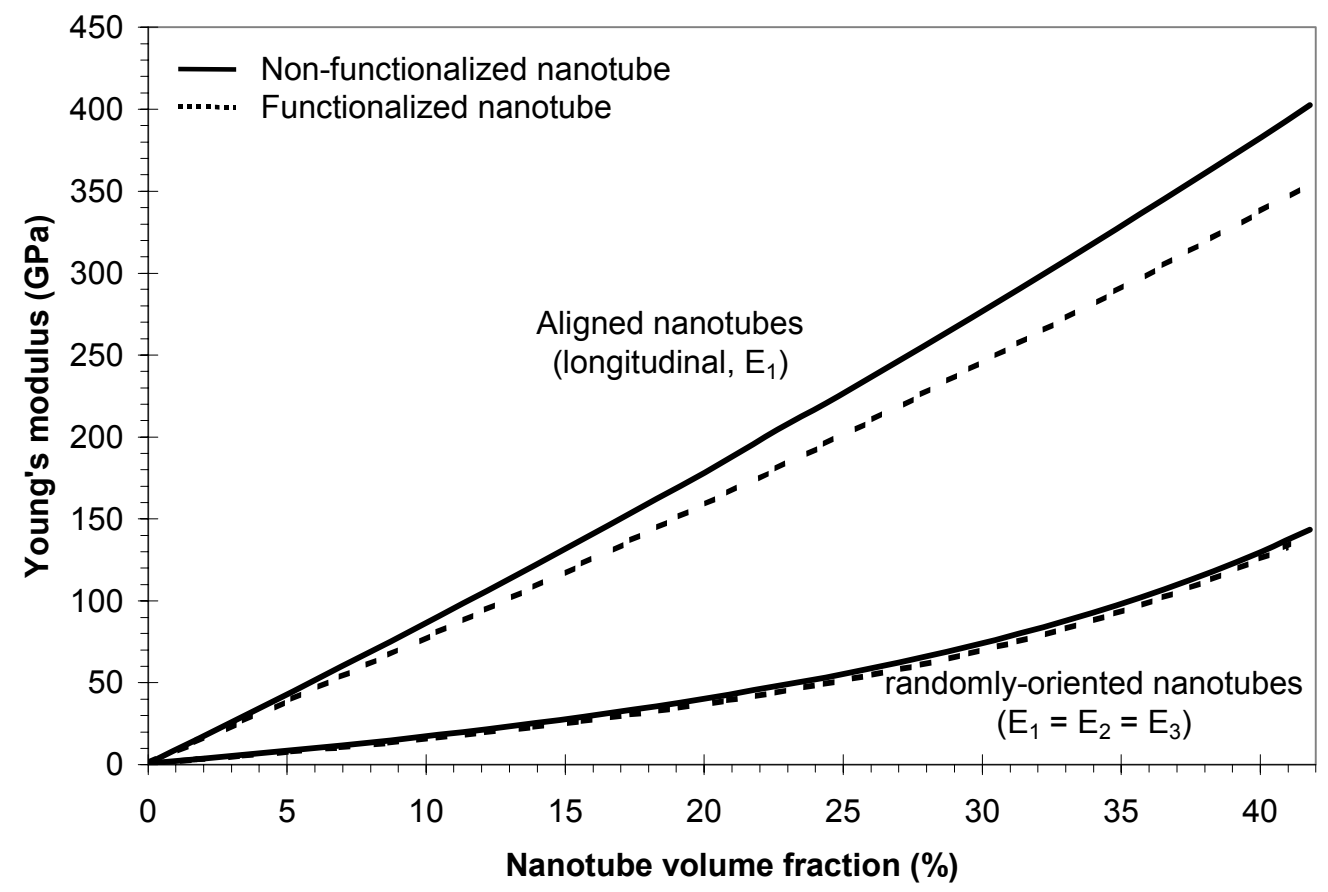

Figure 6. Longitudinal Young's moduli of both composite systems vs. nanotube volume fraction for a nanotube length of $400 \mathrm{~nm}$ 


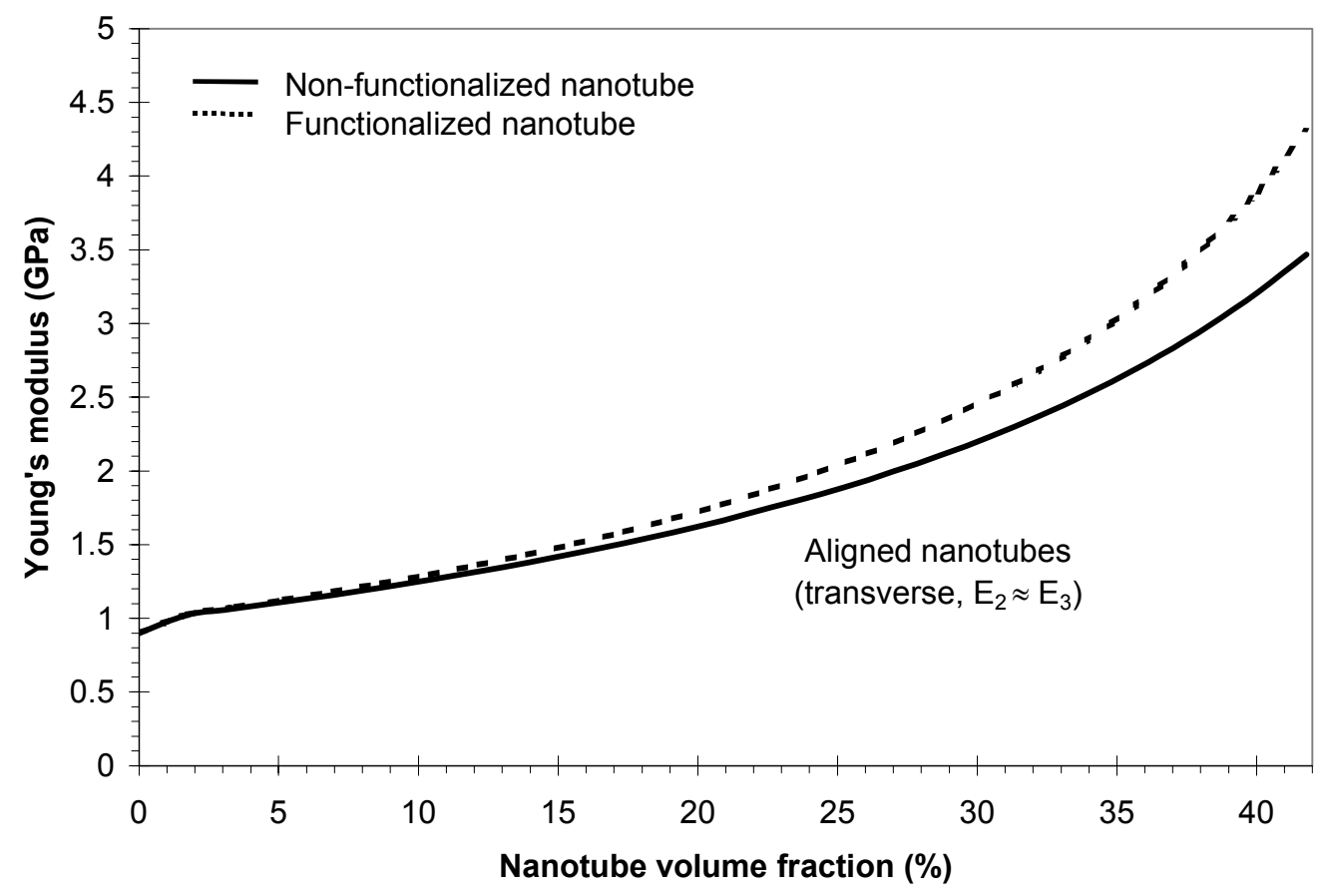

Figure 7. Transverse Young's moduli of both composite systems vs. nanotube volume fraction for a nanotube length of $400 \mathrm{~nm}$

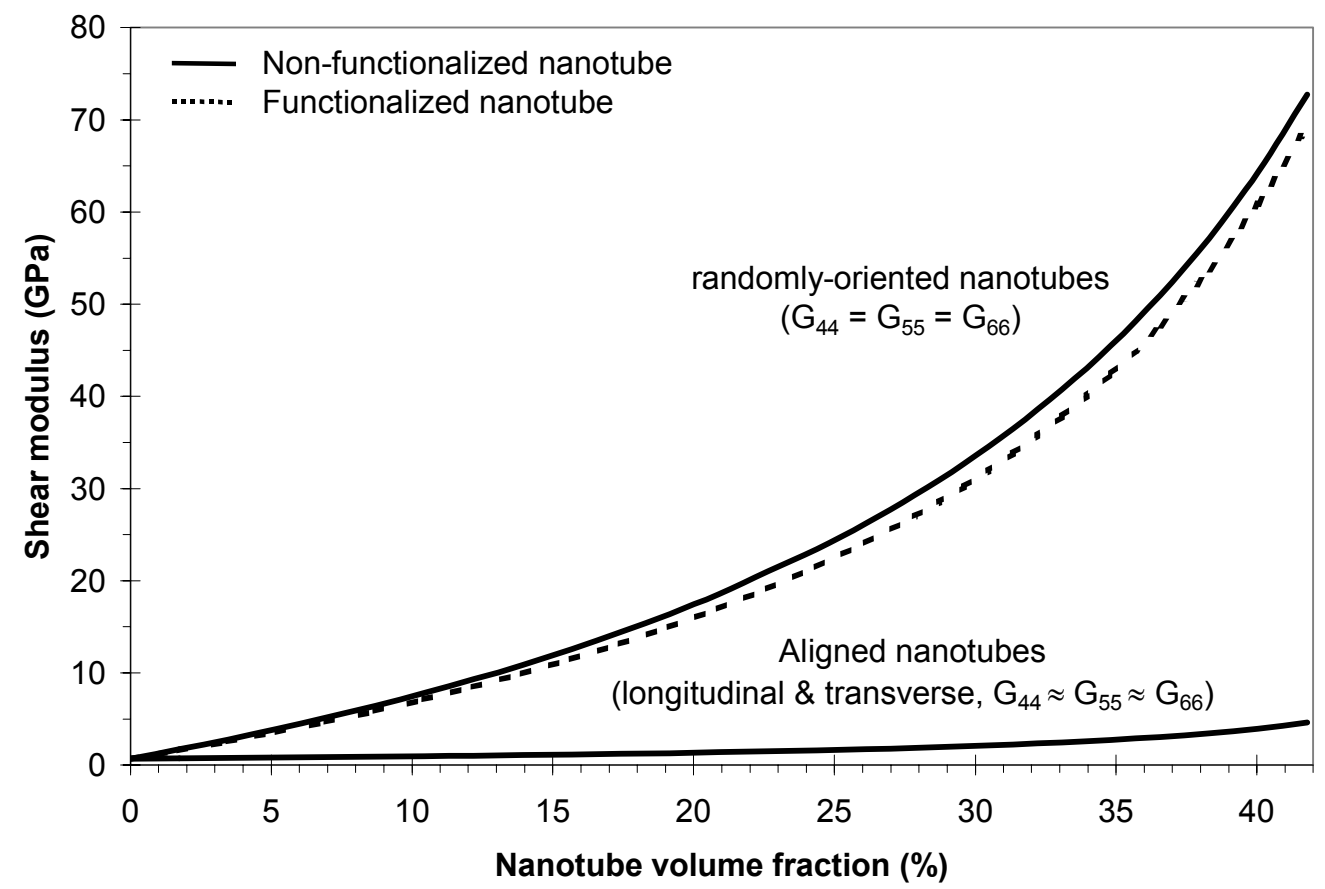

Figure 8. Shear moduli of both composite systems vs. nanotube volume fraction for a nanotube length of $400 \mathrm{~nm}$ 